\title{
Letters to Analytical Chemistry
}

\section{Metal Cation Mediated-Capillary Electrophoresis of Nucleic Acids}

\author{
Tao Li, Dapeng Zhang, Wenru Luo, Meiling Lu, Zhixin Wang, Yuling Song, and Hailin Wang* \\ State Key Laboratory of Environmental Chemistry and Ecotoxicology, Research Center For Eco-Environmental \\ Sciences, Chinese Academy of Sciences, Shuangqing Road 18, Beijing 100085, China
}

Nucleic acid electrophoresis separation heavily depends upon gel or nongel sieving matrix. Here we propose a metal ion mediated-capillary electrophoresis (MCE-CE) by utilizing the nonspecific interactions of $\mathrm{Mg}^{2+}$ and $\mathrm{Ca}^{2+}$ and demonstrate the size, conformation, or sequence based separation and characterization of versatile nucleic acid molecules in free solution. $\mathrm{Mg}^{2+}$ and $\mathrm{Ca}^{2+}$ can induce DNA separation at the concentrations as low as 100 and $50 \mu \mathrm{M}$, respectively. Noteworthy, the two naturally occurring polymorphisms of one base substitution that may change the secondary folding structure or base stacking can be discriminated by MCM-CE, showing its unique capability of resolving length-identical but conformation-different ssDNA. Benefiting from the achieved separation, we further demonstrate that the folding conformation of oligomers and its change caused by single base substitution can be promptly sensed by online coupled fluorescence polarization. We anticipate that this method will be applicable in length polymorphism analysis, singlestrand polymorphism analysis, hybridization analysis, microRNA analysis, and study of protein-nucleic acid interactions and the conformation-function relationship.

Beyond the well-known coding of inheritable information by the primary structure of nucleic acids, recent landmark discoveries reveal that versatile nucleic acid molecules function as regulation of gene expression (RNA interference, microRNA, riboswitch, DNA methylation), recognition of specific substrates (aptamer), and/or catalysis (DNAzyme, ribozyme, aptazyme), which may be underlined by their secondary or tertiary structures. ${ }^{1}$ Although sieving matrix-dependent capillary electrophoresis can provide high throughput and accurate sequencing for the primary structure and methylation of DNA now, ${ }^{2-5}$ there is a lack of an efficient separation means to identify and characterize complex secondary

\footnotetext{
* To whom correspondence should be addressed. E-mail: hlwang@rcees.ac.cn. (1) Hecht, S. M. J. Am. Chem. Soc. 2008, 130, 6859-6866.

(2) Klepárnik, K.; Boček, P. Chem. Rev. 2007, 107, 5279-5317.

(3) Collins, F. S.; Lander, E. S.; Rogers, J.; Waterston, R. H. Nature 2004, 431, 931-945.

(4) Dovichi, N. J.; Zhang, J. Angew. Chem., Int. Ed. 2000, 39, 4463-4468.

(5) Voss, K. O.; Roos, K. P.; Nonay, R. L.; Dovichi, N. J. Anal. Chem. 1998, $70,3818-3823$.
}

and tertiary structures of nucleic acids in free solution. In traditional electrophoresis (as gel or nongel sieving electrophoresis), nucleic acid molecules are often required to interact with the polymer based sieving matrix, ${ }^{6,7}$ which may interfere with their native conformations. Free solution capillary electrophoresis is ideal for conformational separation and analysis because of the preservation of native conformations of nucleic acids. Although nucleic acids are negatively charged, the separation of oligonucleotides in free solution was only observed up to 10 bases because of the well-known small difference in electrophoretic mobility for nucleic acids as the base number increases. ${ }^{8-11}$ The sizeseparation may be achieved through the modification of DNA by a neutral drag-tag polymer ${ }^{12}$ or DNA binding proteins. ${ }^{13,14}$ With the use of a buffer system containing zinc and sodium dodecyl sulfate (SDS) micelle, it is possible to separate 14 out of 18 oligonucleotides of 8 bases. ${ }^{8}$ The specific interactions of metal ions and oligonucleotides (e.g., $\mathrm{K}^{+}$and G-quadruplex and $\mathrm{Ni}^{2+}$ and guanosine) were also used to separate short oligonucleotides. However, these separations were limited to the oligonucleotides of shorter than 21 bases. ${ }^{8,11,15,16}$ It is evident that these separation techniques cannot be combined with the widely applicable polymerase chain reaction (PCR) amplification techniques, which involves two primers of about 20 bases. Therefore, the practical application of these techniques is limited. Here, we propose a metal cation mediated-capillary electrophoresis (MCM$\mathrm{CE}$ ) and demonstrate a free solution separation of versatile nucleic acids by utilizing the nonspecific interactions of $\mathrm{Mg}^{2+}$ and $\mathrm{Ca}^{2+}$. The method does not involve any conformation interfering

(6) Litos, I. K.; Ioannou, P. C.; Christtopoulos, T. K.; Traeger-Synodinos, J.; Kanavakis, E. Anal. Chem. 2007, 79, 395-402.

(7) Atha, D. H.; Kasprzak, W.; O'Connell, C. D.; Shapiro, B. A. Nucleic Acids Res. 2001, 29, 4643-4653.

(8) Cohen, A. s.; Terabe, S.; Smith, J. A.; Karger, B. L. Anal. Chem. 1987, 59, 1021-1027.

(9) Viovy, J.-L. Rev. Mod. Phys. 2000, 72, 813-872.

(10) Slater, G. W.; Desruisseaux, C.; Hubert, S. J.; Mercier, J. F.; Labrie, J.; Boileau, J.; Tessier, F.; Pépin, M. P. Electrophoresis 2000, 21, 3873-3887.

(11) Stellwagen, E.; Stellwagen, N. C. Electrophoresis 2002, 23, 2794-2803.

(12) Vreeland, W. N.; Meagher, R. J.; Barron, A. E. Anal. Chem. 2002, 74, 43284333.

(13) Berezovski, A. P.; Krylov, S. N. J. Am. Chem. Soc. 2003, 125, 13451-13454.

(14) Berezovski, A. P.; Krylov, S. N. Anal. Chem. 2006, 78, 2035-2038.

(15) Huang, C.-C.; Cao, Z.; Chang, H.-T.; Tang, W. Anal. Chem. 2004, 76, 69736981.

(16) Takahashi, T.; Sakurai, T.; Hirata, K.; Hoshino, H. Analyst 2009, 134, 12991301.

Analytical Chemistry, Vol. 82, No. 2, January 15, 2010

487 


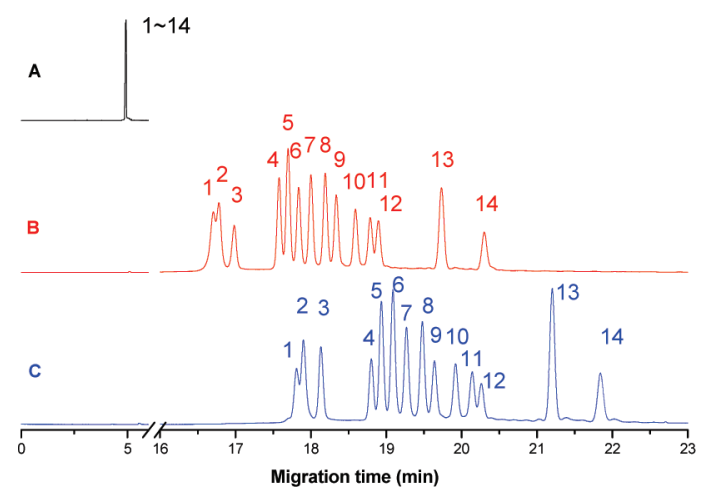

Figure 1. Separation of 14 single stranded poly $\mathrm{dT}$ oligomers by MCM-CE without metal cation (A) and with $2 \mathrm{mM} \mathrm{MgCl}_{2}$ (B) or $2 \mathrm{mM}$ $\mathrm{CaCl}_{2}$ (C). Peaks 1-14 correspond to poly(dT) of 90mer, 80mer, $63 \mathrm{mer}$, 38mer, 35mer, 32mer, 29mer, $26 \mathrm{mer}$, $24 \mathrm{mer}$, $21 \mathrm{mer}$, 19mer, $18 \mathrm{mer}, 12 \mathrm{mer}$, and $9 \mathrm{mer}$. Tetramethylrhodamine was labeled at the $5^{\prime}$ end of each oligomer for online laser-induced fluorescence detection.

sieving-matrix and provides exquisite characterization of function associated conformations of nucleic acids that is a new discovery realm for the study of nucleic acids now. ${ }^{1}$

First, we demonstrate a size-dependent separation by MCMCE. In this case, 14 poly (dT) oligomers with a length of 9-90 bases are chosen because they do not have any secondary folding structure and have weak base stacking. In the absence of metal cation in the run buffer $(25 \mathrm{mM}$ Tris and $192 \mathrm{mM}$ glycine, $\mathrm{pH}$ 8.3), the mixture of 14 poly(dT) overlap as one peak in spite of an apparent size-difference (Figure 1A). Interestingly, 14 poly(dT) can be well separated within $21 \mathrm{~min}$ in the presence of $2 \mathrm{mM}$ $\mathrm{Mg}^{2+}$ and migrated out in the order of the reducing length (Figure 1B). Each peak was identified by injection of single pure oligomer and validated by denaturing gel electrophoresis (Figure S1 in the Supporting Information). One-base separation resolution can be achieved for the two oligomers of $\mathrm{dT}_{18}$ and $\mathrm{dT}_{19}$ (Figure $1 \mathrm{~B}$, peaks 11 and 12). The separation efficiency of 14 poly(dT) oligomers ranged from $2.3 \times 10^{5}$ to $5.2 \times 10^{5}$ theoretical plates. This was achieved by the use of an uncoated fused-silica capillary with an inner diameter of $25 \mu \mathrm{m}$ and an effective length of $43.5 \mathrm{~cm}$. By the use of a capillary of narrower diameter (10 $\mu \mathrm{m}$, i.d.) but the same length, a higher separation efficiency can be obtained $\left(4.0 \times 10^{5} \sim 8.7 \times 10^{5}\right.$ theoretical plates $)$. The observation of size-dependent electrophoresis separation of DNA suggests that $\mathrm{Mg}^{2+}$ can mediate electrophoretic mobility in a size-dependent manner by partial charge neutralization. By estimation of the apparent electrophoretic mobility, $\mathrm{dT}_{9}$ and $\mathrm{dT}_{90}$ have the same value without involvement of $\mathrm{Mg}^{2+}$. With the involvement of $2 \mathrm{mM} \mathrm{Mg}^{2+}, \mathrm{dT}_{9}$ and $\mathrm{dT}_{90}$ have a $20.8 \%$ difference in the apparent mobility (Figure S2 in the Supporting Information). By highly efficient $\mathrm{CE}$ separation, such a moderate difference in mobility change can bring up a successful separation of 14 poly (dT) oligomers. Similar separation for the 14 poly(dT) oligomers can also be achieved by the use of $2 \mathrm{mM} \mathrm{Ca}^{2+}$ (Figure 1C). It is interesting that $\mathrm{Mg}^{2+}$ and $\mathrm{Ca}^{2+}$ at low concentration (e.g., $100 \mu \mathrm{M} \mathrm{Mg}^{2+}$ or $50 \mu \mathrm{M} \mathrm{Ca}^{2+}$ ) can also induce DNA separation (data not shown).

The successful size-dependent separation may allow for hybridization analysis, which is widely applied for detection of a specific sequence in genome, mRNA, and microRNA. As shown

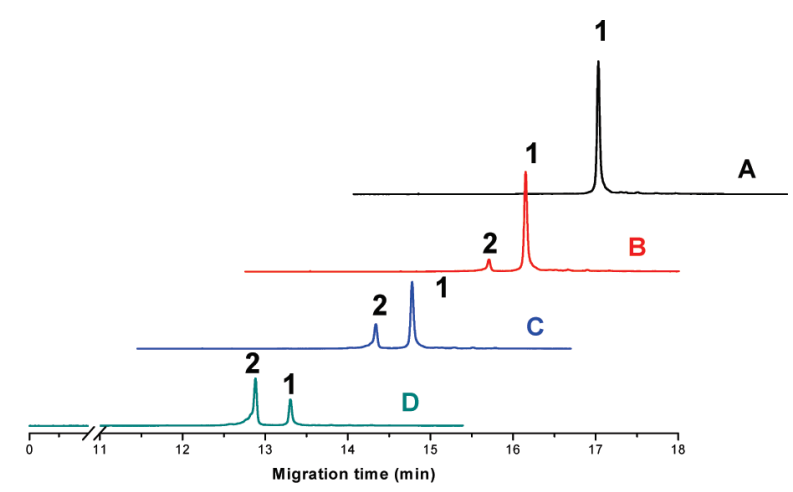

Figure 2. MCM-CE based hybridization analysis. The TMR-ss37mer (peak 1) was titrated by the unlabeled complementary ss 37 mer of 0 (A), $1.45 \times 10^{-18} \mathrm{~mol}(B), 2.9 \times 10^{-18} \mathrm{~mol}(\mathrm{C})$, and $5.8 \times 10^{-18} \mathrm{~mol}$ (D). Peak 2 is the TMR-ds 37 mer. The run buffer contained $5 \mathrm{mM}$ $\mathrm{Mg}^{2+}$.

in Figure 2, with the involvement of $5 \mathrm{mM} \mathrm{Mg}^{2+}$, the ss37mer and ds37mer that was formed through a rapid hybridization reaction can be well separated within $14 \mathrm{~min}$. The low limit of detection for the hybridization analysis based on MCM-CE coupled laser-induced fluorescence (LIF) was estimated as 9.5 $\times 10^{-21} \mathrm{~mol}$ by the ratio of signal-to-noise $=3$. Compared with gel and nongel sieving electrophoresis, the sensitivity is greatly enhanced because of the no use of sieving matrix, which can cause strong light scattering and increase background noise when excited by laser light.

We further demonstrate a separation of length-identical but conformation-different ssDNA by MCM-CE. A naturally occurring polymorphism of p53 (codon $72 \mathrm{Arg} / \mathrm{Pro}$ ) was used for an example, and a 63-mer with a single-base change $(\mathrm{C} \rightarrow \mathrm{G})$ in the middle (p53-1, p53-2, see Table S1 in the Supporting Information) was designed according to previous work. ${ }^{17}$ This base substitution changes the secondary structure and its folding free energy from $-5.7 \mathrm{kcal} / \mathrm{mol}$ (p53-1) to $-7.5 \mathrm{kcal} / \mathrm{mol}$ (p53-2). The stable secondary folding structures of the oligomers that were simulated by the software DNAMAN were shown in the insert of Figure $3 \mathrm{~B}$. In the absence of any metal cation in the run buffer, the three 63 mers (p53-1, p53-2, and $\mathrm{dT}_{63}$ ) were completely overlapped as one peak (Figure 3A). Furthermore, the oligomers of p53-1 and p53-2 cannot be resolved by native polyacrylamide gel electrophoresis (PAGE), denaturing PAGE, and even DNA sequencing PAGE (Figure S3 in the Supporting Information). It is interesting that the two p53 oligomers (p53-1 and p53-2) were well separated from length-identical and secondary structure-absent $\mathrm{dT}_{63}$ and also well separated from each other by involvement with $2 \mathrm{mM} \mathrm{Ca}^{2+}$ (Figure 3B).

Interestingly, two oligomers (HBB-1 and HBB-2, see Table S1 in the Supporting Information) with a single base change of $\mathrm{A} \rightarrow$ $\mathrm{T}$, which does not cause any folding free energy change but alters the base stacking of nine neighbored purines, can also be discriminated by MCM-CE (Figure 4B). The single base substitution occurs in the $\beta$-hemoglobin subunit and causes sickle cell anemia. ${ }^{17}$ Similarly, the two oligomers are unresolvable by free solution CE in the absence of metal cation (Figure 4A) and by native PAGE, denaturing PAGE, and DNA sequencing PAGE

(17) Bar-Ziv, R.; Libchaber, A. Proc. Natl. Acad. Sci. U.S.A. 2001, 98, 90689073. 


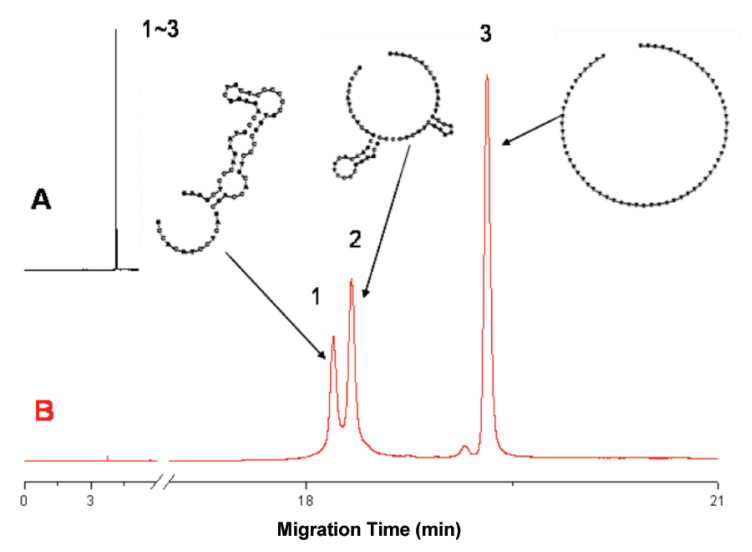

Figure 3. MCM-CE separation of two p53 fragments with a singlebase substitution without metal cation (A) or with $2 \mathrm{mM} \mathrm{CaCl}_{2}(B)$. Peak 1-3 are p53-1, p53-2, and poly $(\mathrm{dT})_{63}$. The oligomers were tagged by TMR for online laser-induced fluorescence detection.

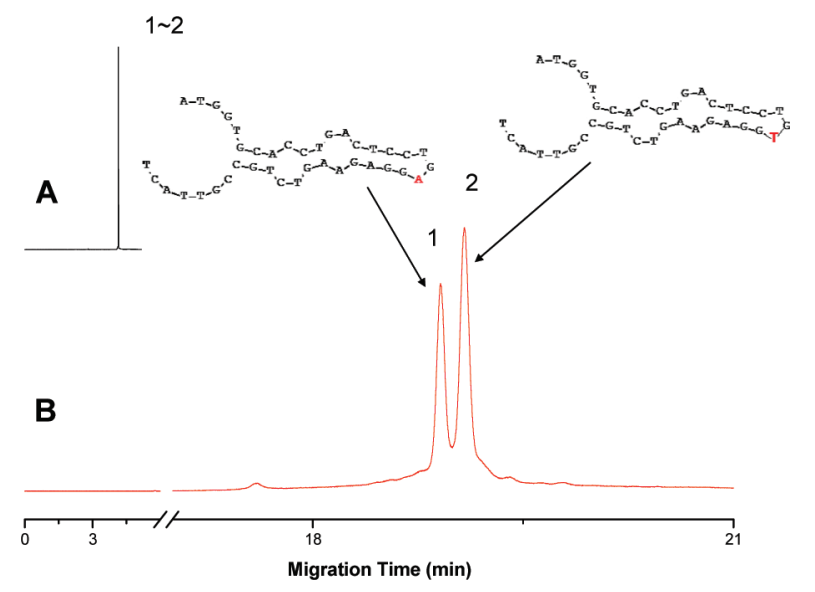

Figure 4. MCM-CE separation of a polymorphism of sickle cell anemia related $\beta$-hemoglobin with a single-base change in the absence of metal cation (A) or the presence of $2 \mathrm{mM} \mathrm{CaCl}_{2}(B)$. Peak identity: 1, HBB-1; 2, HBB-2. The oligomers were tagged by Сy3 for online laser-induced fluorescence detection.

(Figure S4 in the Supporting Information). In addition, the bands of the two unresolved oligomers become smeared on $15 \%$ native PAGE, indicating the occurrence of physiologically irrelevant interaction of the oligomers with the gel network structure, which may interfere with the native conformation of the oligomers. In contrast, the two HBB oligomers can be well separated as two single and narrow peaks by MCM-CE involving $2 \mathrm{mM} \mathrm{Ca}^{2+}$ (Figure 4B). The MCM-CE does not require any gel and eliminates the interaction of nucleic acids with gel network structure, favoring characterization of their native conformations.

With the benefit from the achieved separation, the solution conformation of the oligomers with single base substitution can be further characterized by laser-induced fluorescence polarization coupled with $\mathrm{CE}$. The measured polarization values for the oligomers of p53-1 and p53-2 are 0.217 \pm 0.004 and $0.231 \pm 0.005$ $(\mathrm{n}=3)$, respectively. These large polarization values may result from the folding conformations of the oligomers (Figure 3, insert). In contrast, $\mathrm{dT}_{63}$ with the same length but no folding conformation displays a much lower polarization value $(\mathrm{P}=0.069 \pm$ 0.006). The association of the large polarization of the oligomers

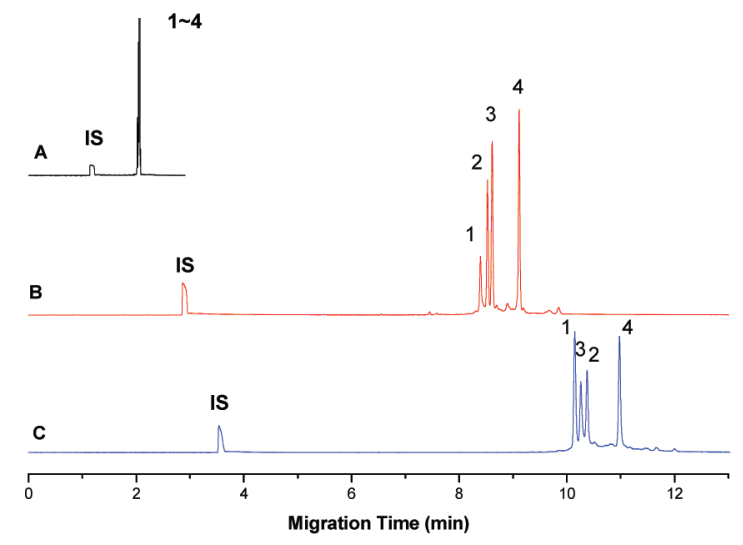

Figure 5. MCM-CE separation of four HIV-1 related microRNAs: (A) without any metal ions, (B) $5 \mathrm{mM} \mathrm{MgCl}_{2}$, and (C) $5 \mathrm{mM} \mathrm{CaCl}_{2}$. Peak identity: 1, has-miR-150; 2, has-miR-125b; 3, has-miR-382; 4, has-miR-223. IS represents the electroosmotic flow marker. The microRNAs were tagged by Cy3 for online laser-induced fluorescence detection.

with a folding conformation is consistent with recent work. ${ }^{18,19}$ The results suggest that the folding conformation can be sensed by fluorescence polarization measurement. Exquisitely, the difference in the folding conformation of the two oligomers (Figure 3 , insert) caused by single base change of $\mathrm{C} \rightarrow \mathrm{G}$ can also be distinguished by fluorescence polarization $(P=0.217$ for p53-1 and 0.231 for p53-2). The oligomers of HBB-1 and HBB-2 with altered base stacking but no computable change in secondary structure have the same polarization value $(P=0.231 \pm 0.012)$, further proving that the folded conformation can be sensed by fluorescence polarization.

By taking advantage of the unique separation capacity of MCM$\mathrm{CE}$, we examined the separation of length-identical but sequence different microRNAs. MicroRNAs are small regulatory RNA molecules that are predicted to function as a modulating expression of $\sim 90 \%$ of all human genes and are important in a wide range of physiologic and pathologic processes. ${ }^{20-23}$ Astonishingly, such a multitude of human microRNA molecules (600-1000) are distributed in an extremely narrow size range (18-24 nucleotides $(\mathrm{nt})) .{ }^{24}$ There is no method reported for separation of natural microRNA. Here we used four HIV-I related microRNAs (hasmiR-150, has-miR-382, has-miR-125b, and has-miR-223) as an example. All the four microRNAs are $22 \mathrm{nt}$ long with different sequences. These microRNAs were labeled by a fluorescent dye Cy3 to allow for on-column laser induced fluorescence detection. The separation of the four microRNAs in free solution $\mathrm{CE}$ in the absence of any metal ion is poor (Figure 5A). However, they can be well separated by using $\mathrm{Ca}^{2+}$ and $\mathrm{Mg}^{2+}$ (Figure 5B). It is interesting that the migration order of the two microRNAs (hasmiR-125b and has-miR-382) by $\mathrm{Mg}^{2+}$ is different from that by

(18) Wang, H.; Lu, M.; Tang, M.-S.; Houten, B. V.; Alexander Ross, J. B.; Weinfeld, M.; Le, X. C. Proc. Natl. Acad. Sci. U.S.A. 2009, 106, 1284912854.

(19) Ruta, J.; Perrier, S.; Ravelet, C.; Fize, J.; Peyrin, E. Anal. Chem. 2009, 81, 7468-7473.

(20) Bartel, P. Cell 2004, 116, 281-297.

(21) Stefani, G.; Slack, F. J. Nat. Rev. Mol. Cell Biol. 2008, 9, 219-230.

(22) Bartel, D. P. Cell 2004, 116, 281-297.

(23) Vasudevan, S.; Tong, Y.; Steitz, J. A. Science 2007, 318, 1931-1934.

(24) Bentwich, I.; Avniel, A.; Karov, Y.; Aharonov, R.; Gilad, S.; Barad, O.; Barzilai, A.; Einat, P.; Einav, U.; Meiri, E.; Sharon, E.; Spector, Y.; Bentwich, Z. Nat. Genet. 2005, 37, 766-770. 
$\mathrm{Ca}^{2+}$. The change in migration order of the microRNAs may indicate that subtle separation selectivity can be provided by using different metal ions.

Here we demonstrate the separation and characterization of versatile nucleic acid molecules in terms of size, conformation, and sequence by $\mathrm{Mg}^{2+}$ and $\mathrm{Ca}^{2+}$ based MCM-CE. It has long been thought that metal ions may deteriorate electrophoresis separation $^{8,25}$ because of causing significantly elevated Joule heating and band broadening. This study established an entirely different concept about the roles of metal ions being played in nucleic acids separation. The divalent metal ions $\mathrm{Mg}^{2+}$ and $\mathrm{Ca}^{2+}$ may form complexes with either the phosphate groups and/ or bases of the oligonucleotides, ${ }^{8}$ partially neutralizing the negative charges of the oligonucleotides. Such partial neutralization may depend upon the length, sequence, and conformation of the oligonucleotides and, thereby, provide the size, sequence, and conformation-dependent separation of the oligonucleotides. However, the exact mechanism remains unclear because of the complexity of the interactions of metal ions and nucleic acids.

Because of the elimination of troublesome sieving matrix, MCM-CE displays several advantages over gel or nongel sieving capillary electrophoresis: simplicity, low viscosity, and high sensitivity. By using MCM-CE, we can detect $9.5 \times 10^{-21} \mathrm{~mol}$ of oligomer in hybridization analysis. It expects that MCM-CE should be able to extend to on-chip electrophoresis too.

It is the first time to demonstrate the discrimination of singlebase substitutions of ssDNA with a length of longer than 20 bases by free solution based analytical technology. This unique feature may allow for promising applications of MCM-CE in characterization and analysis of various small DNA/RNA molecules, including functional aptamer, ${ }^{26}$ aptazyme, DNAzyme, ribozyme, microRNA, and small noncoding RNA. These DNA/RNA molecules have specific secondary or tertiary structures that may be associated with their regulation, recognition, and catalysis functions. MCM$\mathrm{CE}$ will be applicable in length polymorphism analysis, singlestrand polymorphism analysis, hybridization analysis, and the study of protein-nucleic acid interactions and conformationfunction relationship.

(25) Ruiz-Marie, M. C.; Salas-Solano, O.; Carrilho, E.; Kotler, L.; Karger, B. L. Anal. Chem. 2008, 80, 8598-8604.

(26) Tuerk, C.; Gold, L. Science 1990, 249, 505-510.

\section{EXPERIMENTAL SECTION}

Oligonucleotides. Single stranded oligonucleotides were synthesized by Shanghai Sangon Biological Engineering Technology \& Services Co., Ltd. (Shanghai, China). HIV related microRNAs were synthesized (RNase free HPLC grade) by IDT (Coralville, IA). These oligonucleotides were labeled at the $5^{\prime}$ end by a tetramethylrhodamine (TMR) or cyanine 3 (Cy3) for highly sensitive laser-induced fluorescence detection. The sequence and label of the DNAs and microRNAs were listed in Table S1 in the Supporting Information.

Capillary Electrophoresis. Capillary electrophoresis analyses were performed using a laboratory-built CE-LIF instrument as described recently. ${ }^{18} \mathrm{~A} 543.5 \mathrm{~nm}$ laser beam was utilized to excite fluorescent dye tagged on oligonucleotides, and the fluorescence was detected at $580 \mathrm{~nm}$. Uncoated fused-silica capillaries of 50 cm length $\times 25 \mu$ m i.d. $\times 365 \mu$ m o.d. (effective length, $43.5 \mathrm{~cm}$ ) were used for DNA separation and of $40 \mathrm{~cm}$ length $\times 20 \mu \mathrm{m}$ i.d. $\times 365 \mu$ m o.d. (effective length, $33.5 \mathrm{~cm}$ ) were used for microRNA separation. The run buffer was $25 \mathrm{mM}$ Tris and $192 \mathrm{mM}$ glycine at $\mathrm{pH} 8.3$ with the desired concentrations of $\mathrm{MgCl}_{2}$ or $\mathrm{CaCl}_{2}$. The oligonucleotides were diluted with $20 \mathrm{mM}$ Tris- $\mathrm{HCl}$ buffer $(\mathrm{pH}$ 7.4) that contained $10 \mathrm{mM} \mathrm{Mg}^{2+}$ or $\mathrm{Ca}^{2+}$. The final concentration of each oligonucleotide in sample was $2 \mathrm{nM}$. The samples were electrokinetically injected into the capillary by applying an electric field of $300 \mathrm{~V} / \mathrm{cm}$ for $5 \mathrm{~s}$ and separated by applying an electric field of $400 \mathrm{~V} / \mathrm{cm}$.

\section{ACKNOWLEDGMENT}

This work was supported by the grants from the National Natural Science Foundation of China (Grants 20877091, 20890112, and 20621703), the National Basic Program of China (Grants 2007CB407305 and 09CB421605), and the Chinese Academy of Sciences (Grant YZ200749) to Dr. Hailin Wang.

\section{SUPPORTING INFORMATION AVAILABLE}

Additional information as noted in text. This material is available free of charge via the Internet at http://pubs.acs.org.

Received for review November 10, 2009. Accepted December 13, 2009.

AC9025708 